\title{
Práticas profissionais em saúde do trabalhador na Atenção Primária: desafios para implementação de políticas públicas
}

\author{
Professional practices in occupational health in Primary Care: \\ challenges for the implementation of public policies
}

Deiviane Pereira da Silva (https://orcid.org/0000-0003-0243-1878) ${ }^{1}$

Ronilson Ferreira Freitas (https://orcid.org/0000-0001-9592-1774) ${ }^{2}$

Lucas Faustino de Souza (https://orcid.org/0000-0003-2565-1080) ${ }^{3}$

Nadine Antunes Teixeira (https://orcid.org/0000-0001-7875-2921) ${ }^{3}$

Elizabeth Costa Dias (https://orcid.org/0000-0003-1708-1014) ${ }^{4}$

Josiane Santos Brant Rocha (https://orcid.org/0000-0002-7317-3880) ${ }^{1}$

\footnotetext{
${ }^{1}$ Programa de PósGraduação em Cuidados Primários em Saúde, Universidade Estadual de Montes Claros. Av. Osmani Barbosa. 39404-549 Montes Claros MG Brasil. deivianesilva@yahoo.com.br ${ }^{2}$ Programa de PósGraduação em Saúde, Sociedade e Ambiente, Universidade Federal dos Vales do Jequitinhonha e Mucuri. Diamantina MG Brasil.

${ }^{3}$ Centro Universitário Funorte. Montes Claros MG Brasil.

${ }^{4}$ Faculdade de Medicina, Departamento de Medicina Preventiva e Social, Universidade Federal de Minas Gerais, Belo Horizonte MG Brasil.
}

\begin{abstract}
The actions in Occupational Health developed by Primary Health Care teams in a medium-sized municipality were assessed in a cross-sectional analytical study. A questionnaire consisting of 35 questions, divided up into four sections and answered by 289 health professionals, was used. The results were analyzed according to the distribution of absolute and relative frequencies using the chi-square test with a significance level of 0.05 . The results revealed that activities developed in the area, as well as the epidemiological profile of the working population living in the area of coverage, are acknowledged as being productive, however interventions based on this diagnosis were not identified. It was observed that, among the professionals, more frequently it is the physician who identifies the epidemiological profile of the workers, while the nurse takes the lead role in the surveillance of diseases and in the technical-pedagogical support for the development of actions in Occupational Health. Based on the findings, it was observed that teams find it difficult to incorporate actions in Occupational Health in the planning of daily activities in their praxis, suggesting the need for professional qualification and pedagogical and institutional technical support from the specialized units.

Key words Occupational health, Primary health care, Surveillance in occupational health
\end{abstract}

Resumo Foram analisadas as ações de Saúde do Trabalhador desenvolvidas pelas equipes da Atenção Primária em município de médio porte em estudo transversal analítico. Utilizou-se questionário contendo 35 questões, distribuídas em quatro blocos, respondidas por 289 profissionais de saúde. Os resultados foram analisados segundo a distribuição das frequências absolutas e relativas com o teste de qui-quadrado, ao nível de significância de 0,05. Os resultados demonstraram que são reconhecidas as atividades produtivas desenvolvidas no território assim como o perfil epidemiológico da população trabalhadora residente em sua área de abrangência, entretanto, não foram identificadas intervenções orientadas por esse diagnóstico. Observou-se que, entre os profissionais, o médico identifica com mais frequência o perfil epidemiológico dos trabalhadores e o enfermeiro destaca-se na vigilância dos agravos e no apoio técnico-pedagógico para o desenvolvimento de ações de Saúde do Trabalhador. Com base nos achados, observouse que as equipes têm dificuldade para incorporar as ações de Saúde do Trabalhador no planejamento das atividades cotidianas de sua práxis, sugerindo a necessidade de qualificação profissional e suporte técnico pedagógico e institucional pelas instâncias especializadas.

Palavras-chave Saúde do trabalhador, Atenção Primária à Saúde, Vigilância em Saúde do Trabalhador 


\section{Introdução}

A Saúde do Trabalhador (ST) configura-se como um campo de atuação do Sistema Único de Saúde (SUS) que tem por finalidade intervir sobre o processo saúde-doença dos trabalhadores, de modo a garantir uma atenção integral que incorpore práticas de promoção e proteção da saúde, bem como vigilância, assistência e reabilitação ${ }^{1}$, considerando o trabalho como determinante das condições de vida e de saúde da população².

A publicação da Política Nacional de Saúde do Trabalhador e da Trabalhadora (PNSTT) em 2012 reforçou a importância de propiciar o cuidado integral aos trabalhadores, o que se inicia na Atenção Primária à Saúde (APS) ${ }^{1}$, favorecendo o acesso dos trabalhadores à assistência à saúde, com qualidade, resolutividade e com capacidade para compreender os modos como o trabalho interfere nas suas condições de vida e saúde, bem como a implementação das ações de vigilância ${ }^{3}$.

Entretanto, apesar dos avanços observados nos últimos 30 anos, a inserção de um cuidado qualificado aos trabalhadores no âmbito do SUS mostra-se insuficiente para atender as necessidades impostas pelas transformações no mundo do trabalho, marcadas pelo crescimento da informalidade, a precarização dos vínculos e a domicialização das atividades, que têm consequências dramáticas sobre a saúde e a vida dos trabalhadores ${ }^{4,5}$.

Segundo estimativas da Organização Internacional do Trabalho (OIT) ${ }^{6}$, ocorrem no mundo, anualmente, 2,78 milhões de mortes por causas relacionadas ao trabalho. Destas, cerca de 2,4 milhões são decorrentes de doenças profissionais, enquanto 380 mil se devem aos acidentes 7 . Dados do Observatório Digital de Saúde e Segurança do Trabalho $^{8}$ registram a ocorrência de 3.879 .755 acidentes de trabalho no Brasil entre os anos de 2012 a 2017 em trabalhadores do mercado formal, apontando que os agravos relacionados ao ofício configuram um problema de saúde públi$\mathrm{ca}^{9}$ e colocam o Brasil em quarto lugar, no mundo, em número de acidentes nessa área ${ }^{8}$.

Nesse cenário, o SUS assume particular importância na atenção aos trabalhadores, considerando o panorama de elevados acidentes e doenças relacionadas ao trabalho, registrados nos sistemas oficiais ${ }^{9}$ e reconhecendo a centralidade da APS na assistência à saúde dessas pessoas ${ }^{3}$. Entretanto, para que cumpra bem esse papel, é necessário à APS conhecer melhor o processo de trabalho das equipes, as dificuldades que encontram enquanto trabalhadores e profissionais de saúde para a superação dos entraves. Assim, torna-se necessário ampliar a cooperação entre as instâncias de gestão da APS e a $\mathrm{ST}^{10}$ bem como implementar apoio técnico pedagógico, ou matriciamento das equipes pelas instâncias responsáveis; os Centros de Referência em Saúde do Trabalhador (CEREST), os Núcleos Ampliados de Saúde da Família (NASF) e as áreas de Vigilância em Saúde.

O conhecimento produzido pela literatura acerca das ações em ST, desenvolvidas pelos profissionais da APS, demonstraram que as práticas de ST ainda não estão incorporadas no cotidiano de trabalho das equipes de APS ${ }^{3}$. Dessa forma, as ações de assistência à ST ainda são escassas, pontuais e não acontecem de forma articulada com a PNSTT ${ }^{11-14}$. É possível evidenciar que as ações mais relatadas são o atendimento e as notificações de agravos relacionados ao trabalho (acidentes de trabalho e doenças ocupacionais) e o mapeamento das atividades produtivas ${ }^{3,15}$.

São apontados como fatores dificultadores para a realização das ações de ST são: a sobrecarga de trabalho da equipe, a formação profissional inadequada/despreparo, a falta de apoio institucional; fatores relacionados à Comunicação de Acidentes de Trabalho (CAT), ao nexo causal, à condição de trabalho do funcionário da saúde, à vida do trabalhador e sua relação com o labor e à lógica da produtividade ${ }^{15,16}$. E como fatores facilitadores, destaca-se o apoio matricial pelo CEREST e por outras instâncias do SUS ${ }^{3,14}$.

Nesse contexto, torna-se necessária a busca de apoio para fortalecer a assistência à ST, que deve agrupar recursos metodológicos, com vistas a atender aos objetivos dos gestores, profissionais de saúde e usuários. Quanto a isso, a gestão compartilhada entre os sujeitos é construída a partir de relações horizontalizadas e solidárias entre as equipes de saúde da família e os apoiadores institucionais, respeitando-se os diferentes saberes e práticas, compartilhando dificuldades e construindo estratégias de intervenção ${ }^{17}$.

O estudo buscou conhecer a incorporação dos cuidados aos trabalhadores no cotidiano dos profissionais da APS em município de grande porte do Norte de Minas Gerais, com a finalidade de contribuir para potencializar o desenvolvimento de ações de ST, na perspectiva da integralidade da atenção.

\section{Metodologia}

Trata-se de estudo transversal, analítico, desenvolvido nas unidades de APS do município de 
Montes Claros/MG, o qual é sede de CEREST Regional, habilitado pela Portaria no 102 , de 25 de novembro de $2008^{18}$. Em outubro de 2017, no início da pesquisa, existiam 132 equipes de ESF implantadas, sendo 122 na área urbana e 10 na área rural, evidenciando uma cobertura de cem por cento do município. A população da pesquisa foi constituída pelos cirurgiões-dentistas (CD), enfermeiros e médicos cadastrados nas Unidades Básicas de Saúde do município, que, à época do trabalho de campo, perfaziam um total de 326 trabalhadores, distribuídos em 132 equipes. Incluíram-se neste estudo os profissionais de nível superior, considerando o tipo de assistência prestada na APS. Foram excluídos os profissionais que se encontravam afastados do trabalho por férias, atestado médico ou licença-maternidade.

A coleta de dados ocorreu no período de outubro de 2017 a março de 2018. Inicialmente foi realizado contato prévio com o Núcleo de Atenção Primária à Saúde (NAPRIS) do município de Montes Claros para autorização e planejamento. Após anuência desse núcleo, a aplicação dos questionários ocorreu durante reuniões agendadas, divididas por categoria profissional. Posteriormente, realizaram-se levantamentos e busca pelos profissionais que não estavam presentes nas reuniões, a partir das listas de presença, e procedeu-se à aplicação dos questionários na unidade da qual o profissional fazia parte, com contato telefônico e agendamento prévio.

Utilizou-se questionário adaptado do estudo de Amorim realizado em 2016 intitulado "Vigilância em Saúde do Trabalhador na Atenção Básica: Contribuições para o aprimoramento das ações de saúde no município de João Pessoa - $\mathrm{PB}^{19}$. Trata-se de instrumento autoaplicável, com uso de escala tipo Likert, composto por 35 itens na versão adaptada. O bloco 1 contemplava os dados sociodemográficos; bloco 2 avaliava as ações de atenção à saúde dos trabalhadores desenvolvidas pelas equipes de APS; o bloco 3 analisava as práticas de atenção à saúde dos usuários trabalhadores no âmbito da APS e o bloco 4 investigava o apoio técnico-pedagógico e institucional para o desenvolvimento da atenção à ST no âmbito da APS.

A caracterização da população ocorreu a partir da distribuição das frequências absolutas e percentuais das variáveis analisadas no bloco 1 , segundo categoria profissional. Para a descrição das ações de atenção à saúde dos trabalhadores desenvolvidas pela equipe de APS (bloco 2), realizou-se a recategorização das respostas em ne- gativas (nunca, raramente, às vezes), positivas (quase sempre e sempre) e não sei, as quais foram comparadas às suas frequências e percentuais. Para comparar as frequências absolutas e relativas das práticas de atenção à saúde dos usuários trabalhadores, desenvolvidas pelos profissionais no âmbito da APS (bloco 3) e o apoio para o desenvolvimento da atenção à ST no âmbito da APS (bloco 4) por categoria profissional, as respostas também foram recategorizadas, respectivamente, em negativas (nunca, raramente e às vezes), positivas (quase sempre e sempre), no bloco 3; e negativas (discordo fortemente e discordo) e positivas (concordo e concordo fortemente) no bloco 4.

Previamente à coleta de dados, realizou-se um estudo piloto com profissionais pertencentes às categorias pesquisadas (um cirurgião-dentista, um enfermeiro e um médico) e que já trabalharam anteriormente na APS. Os resultados do estudo piloto possibilitaram o reordenamento dos blocos 2 e 3 do instrumento para apresentar primeiro os aspectos relacionados às ações em ST desenvolvidas pelas equipes de saúde e posteriormente dos profissionais e possibilitou ainda a exclusão da seguinte questão do bloco 3: "A equipe de saúde possui um diagnóstico da situação de saúde dos trabalhadores (perfil epidemiológico) que residem no território de referência de sua unidade?”. Após essa fase, a pesquisa de campo foi iniciada.

Os dados foram tabulados a partir do programa estatístico Statical Package for the Social Science (SPSS), versão 23. Foram apresentados os valores absolutos e relativos, adotando o teste de qui-quadrado, ao nível de significância de 0,05. Para a questão de múltipla escolha do bloco 4 , a análise de dados foi feita a partir da distribuição das frequências absolutas e percentuais.

Após a análise estatística, as questões dos blocos 2 e 3 foram agrupadas em três categorias cada uma. O bloco 2, constituído por dez questões, foi dividido em: atividades produtivas no território (cinco questões), perfil epidemiológico da população adscrita (duas questões) e educação em saúde/controle social (três questões). E o bloco 3, constituído por 15 questões, que foram agrupadas em: identificação de ocupação/riscos e agravos (sete questões), atividades produtivas no território (duas questões) e vigilância de agravos em ST (seis questões).

O estudo foi aprovado pelo Comitê de Ética em Pesquisa da Universidade Estadual de Montes Claros, em setembro de 2017. 


\section{Resultados}

Participaram do estudo 289 (88,6\%) profissionais de saúde, considerando a perda de 37 trabalhadores não respondentes. Daqueles, $61(21,1 \%)$ eram cirurgiões-dentistas, 123 enfermeiros $(42,6 \%)$ e $105(36,3 \%)$ médicos. As características sociodemográficas da população estão descritas na Tabela 1.

$\mathrm{Na}$ caracterização sociodemográfica, houve predominância do sexo feminino $(79,2 \%)$, com a maioria na faixa etária de 31 a 40 anos (48,8\%). Em relação ao tempo de atuação na APS, a maior parte dos CDs $(54,1 \%)$ e enfermeiros $(31,7 \%)$ possuía mais de cinco anos de atuação, e os médicos $(33,3 \%)$, de três a cinco anos. Quanto à formação complementar, a maior parte dos enfermeiros $(49,6 \%)$ fez especialização em saúde da família; os médicos $(43,8 \%)$ e CDs $(47,8 \%)$, residência em saúde da família/medicina, família e comunidade. Apenas $4,5 \%$ da população estudada possuíam formação em ST.

Entre as ações de ST, desenvolvidas pelos profissionais da APS (Tabela 2), predominaram o mapeamento das atividades produtivas desenvolvidas no território da unidade $(59,2 \%)$ e a identificação dos fatores e situações de risco para a saúde dos trabalhadores e da comunidade decorrentes dessas atividades (47,1\%). Entretanto, apenas uma pequena parcela da população estudada declarou discutir sobre a presença desses fatores e situações de risco com a população $(30,8 \%)$, realizar ações para eliminar ou minimizá-los $(21,8 \%)$ e realizar intervenções e ou negociação de medidas de prevenção dos riscos decorrentes das situações de trabalho (22,8 \%).

Quanto ao perfil epidemiológico da população adscrita, observou-se que, em 52,9\% dos casos, o diagnóstico da situação de saúde dos trabalhadores foi considerado e que $58,5 \%$ analisa-

Tabela 1. Caracterização dos sujeitos da pesquisa em número e proporções, estratificadas por categoria profissional, Montes Claros, 2018.

\begin{tabular}{|c|c|c|c|c|}
\hline \multirow{3}{*}{ Variáveis sociodemográficas } & \multicolumn{3}{|c|}{ Categoria Profissional } & \multirow{3}{*}{$\begin{array}{l}\text { Total } \\
\text { n }(\%)\end{array}$} \\
\hline & $\begin{array}{l}\text { Cirurgião- } \\
\text { Dentista }\end{array}$ & Enfermeiro & Médico & \\
\hline & n (\%) & n (\%) & n (\%) & \\
\hline Sexo & $50(82,0)$ & $102(82,9)$ & $77(73,3)$ & $229(79,2)$ \\
\hline \multicolumn{5}{|l|}{ Feminino } \\
\hline Masculino & $11(18)$ & $21(17,1)$ & $28(26,7)$ & $60(20,8)$ \\
\hline \multicolumn{5}{|l|}{ Faixa etária } \\
\hline De 21 a 30 anos & $20(32,8)$ & $51(41,5)$ & $45(42,9)$ & $116(40,1)$ \\
\hline De 31 a 40 anos & $34(55,7)$ & $59(48,0)$ & $48(45,7)$ & $141(48,8)$ \\
\hline De 41 a 50 anos & $06(9,8)$ & $13(10,6)$ & $07(6,7)$ & $26(9,0)$ \\
\hline De 51 a 60 anos & $01(1,6)$ & $00(0,0)$ & $04(3,8)$ & $05(1,7)$ \\
\hline 61 anos ou mais & $00(0,0)$ & $00(0,0)$ & $01(1,0)$ & $01(0,3)$ \\
\hline \multicolumn{5}{|l|}{ Tempo de atuação na APS } \\
\hline Menos de um ano & $05(8,2)$ & $19(15,4)$ & $18(17,1)$ & $42(14,5)$ \\
\hline De um a dois anos & $03(4,9)$ & $31(25,2)$ & $27(25,7)$ & $61(21,1)$ \\
\hline De três a cinco anos & $20(32,8)$ & $34(27,6)$ & $35(33,3)$ & $89(30,8)$ \\
\hline Mais de cinco anos & $33(54,1)$ & $39(31,7)$ & $25(23,8)$ & $97(33,6)$ \\
\hline \multicolumn{5}{|l|}{ Formação complementar } \\
\hline $\begin{array}{l}\text { Especialização em Saúde da } \\
\text { Família. }\end{array}$ & $19(31,1)$ & $61(49,6)$ & $32(30,5)$ & $112(38,8)$ \\
\hline $\begin{array}{l}\text { Residência em Saúde da Família/ } \\
\text { Medicina, Família e Comunidade }\end{array}$ & $29(47,5)$ & $25(20,3)$ & $46(43,8)$ & $100(34,6)$ \\
\hline $\begin{array}{l}\text { Especialização em Saúde do } \\
\text { Trabalhador }\end{array}$ & $01(1,6)$ & $04(3,3)$ & $08(7,6)$ & $13(4,5)$ \\
\hline Mestrado & $03(4,9)$ & $04(3,3)$ & $06(5,7)$ & $13(4,5)$ \\
\hline Doutorado & $02(3,3)$ & $01(0,8)$ & $03(2,9)$ & $06(2,1)$ \\
\hline Outra & $17(27,9)$ & $22(17,9)$ & $09(8,6)$ & $48(16,6)$ \\
\hline Nenhuma & $06(9,8)$ & $10(8,1)$ & $22(21)$ & $38(13,1)$ \\
\hline
\end{tabular}

Fonte: Trabalho de campo realizado no município de Montes Claros, Minas Gerais, Brasil, no período de 2017/2018. 
Tabela 2. Identificação das ações de Saúde do Trabalhador desenvolvidas pela equipe de Atenção Primária à Saúde, 2018.

\begin{tabular}{|c|c|c|c|c|}
\hline \multicolumn{2}{|c|}{ Ações de ST desenvolvidas pela equipe de APS } & Sim & $\begin{array}{l}\text { Não } \\
\text { n (\%) }\end{array}$ & $\begin{array}{l}\text { Não sei } \\
\text { n }(\%)\end{array}$ \\
\hline \multirow{5}{*}{$\begin{array}{l}\text { Atividades } \\
\text { produtivas no } \\
\text { território }\end{array}$} & $\begin{array}{l}\text { Mapeia as atividades produtivas desenvolvidas no } \\
\text { território de referência de sua unidade }\end{array}$ & $171(59,2)$ & $97(33,6)$ & $21(7,3)$ \\
\hline & $\begin{array}{l}\text { Identifica os fatores e situações de risco } \\
\text { decorrentes dessas atividades }\end{array}$ & $136(47,1)$ & $108(37,4)$ & $45(15,6)$ \\
\hline & $\begin{array}{l}\text { Discute com a população sobre a presença de } \\
\text { fatores de risco, decorrentes das atividades } \\
\text { produtivas no território }\end{array}$ & $89(30,8)$ & $182(63,0)$ & $18(6,2)$ \\
\hline & $\begin{array}{l}\text { Realiza alguma ação para eliminar ou minimizar } \\
\text { esses fatores e situações de risco }\end{array}$ & $63(21,8)$ & $212(73,4)$ & $14(4,8)$ \\
\hline & $\begin{array}{l}\text { Realiza ações de intervenção e negociação de } \\
\text { medidas de prevenção dos riscos decorrentes das } \\
\text { situações de trabalho }\end{array}$ & $66(22,8)$ & $201(69,6)$ & $22(7,6)$ \\
\hline \multirow{2}{*}{$\begin{array}{l}\text { Perfil } \\
\text { epidemiológico } \\
\text { da população } \\
\text { adscrita }\end{array}$} & $\begin{array}{l}\text { Possui um diagnóstico da situação de saúde } \\
\text { dos trabalhadores que residem no território de } \\
\text { referência da sua unidade }\end{array}$ & $153(52,9)$ & $136(47,1)$ & $0(0,0)$ \\
\hline & $\begin{array}{l}\text { Analisa a informação decorrente das notificações } \\
\text { de acidentes e doenças relacionadas ao trabalho }\end{array}$ & $169(58,5)$ & $119(41,2)$ & $1(0,3)$ \\
\hline \multirow{3}{*}{$\begin{array}{l}\text { Educação } \\
\text { em Saúde/ } \\
\text { Controle social }\end{array}$} & $\begin{array}{l}\text { Desenvolve ações de educação em saúde do } \\
\text { trabalhador }\end{array}$ & $66(22,8)$ & $\begin{array}{r}212 \\
(73,4)\end{array}$ & $1(3,8)$ \\
\hline & $\begin{array}{l}\text { Promove a mobilização de usuários trabalhadores, } \\
\text { buscando efetivar participação e controle social }\end{array}$ & $68(23,5)$ & $212(73,4)$ & $14(5,5)$ \\
\hline & $\begin{array}{l}\text { Recebe denúncia ou solicitação de intervenção em } \\
\text { situações de risco e/ou adoecimento de usuários e } \\
\text { comunidade provenientes de atividades produtivas }\end{array}$ & $34(11,8)$ & $221(76,5)$ & $34(11,8)$ \\
\hline
\end{tabular}

vam as informações decorrentes das notificações de acidentes e doenças relacionadas ao trabalho. Entretanto, as ações de educação em saúde eram realizadas por uma minoria (22,8\%), assim como as ações de promoção e mobilização de usuários trabalhadores $(23,5 \%)$. Ademais, uma pequena parcela dos profissionais de saúde afirmou receber denúncia ou solicitação de intervenção em situações de risco e/ou adoecimento de usuários e comunidade provenientes de atividades produtivas $(11,8 \%)$.

Ao comparar as práticas de atenção à ST desenvolvidas pelos profissionais de saúde (Tabela 3), observou-se que, em relação à identificação de ocupação/riscos e agravos, a categoria médica demonstrou resultados significativos em relação aos seus grupos de comparação para: perguntar ao usuário quais as atividades de trabalho ele desenvolve na sua ocupação atual $(p=0,001)$, perguntar ao usuário sobre o trabalho/ocupação desenvolvido por ele anteriormente $(p=0,000)$, identificar os riscos para a saúde no trabalho/ocupação pregressa do usuário $(\mathrm{p}=0,004)$ e buscar estabelecer a relação entre a queixa e/ou doença referida pelo usuário com a exposição a situações de risco para a saúde, presentes em seu trabalho/ ocupação, atual ou pregressa $(\mathrm{p}=0,000)$.

Em relação ao desenvolvimento de ações de vigilância dos agravos em ST, observou-se que o enfermeiro obteve resultados significativos em relação às demais categorias para: conhecer o SINAN ( $p=0,000)$, saber que algumas doenças relacionadas ao trabalho são de notificação no SINAN ( $p=0,000)$, notificar os casos de acidente de trabalho no SINAN ( $p=0,000)$, notificar os casos de doenças relacionadas ao trabalho no SINAN $(p=0,000)$ e realizar investigação epidemiológica dos casos suspeitos notificados para confirmação do diagnóstico, fazendo relação com o trabalho $(p=0,000)$. A categoria médica apresentou resultados significativos em relação a elaborar e implantar plano terapêutico e orientar o trabalha- 
Tabela 3. Comparação das práticas de atenção à saúde dos usuários trabalhadores, desenvolvidas pelos profissionais no âmbito da Atenção Primária à Saúde, segundo categoria profissional, 2018.

\begin{tabular}{|c|c|c|c|c|c|c|}
\hline \multirow{2}{*}{\multicolumn{2}{|c|}{$\begin{array}{l}\text { Práticas de atenção à ST desenvolvidas } \\
\text { pelos profissionais de saúde }\end{array}$}} & \multirow{3}{*}{$\begin{array}{c}\begin{array}{c}\text { Cirurgião- } \\
\text { Dentista } \\
(\mathbf{n}=61)\end{array} \\
\text { Sim } \\
\mathbf{n}(\%) \\
\end{array}$} & \multirow{3}{*}{$\begin{array}{c}\begin{array}{c}\text { Enfermeiro } \\
(\mathrm{n}=123)\end{array} \\
\text { Sim } \\
\mathrm{n}(\%) \\
\end{array}$} & \multirow{3}{*}{$\begin{array}{c}\begin{array}{c}\text { Médico } \\
(\mathrm{n}=105)\end{array} \\
\text { Sim } \\
\mathbf{n}(\%) \\
\end{array}$} & \multirow{3}{*}{$\begin{array}{c}\begin{array}{c}\text { Total } \\
(\mathbf{n}=289)\end{array} \\
\text { Sim } \\
\mathbf{n}(\%)\end{array}$} & \multirow[t]{3}{*}{$\mathbf{P}$} \\
\hline & & & & & & \\
\hline & & & & & & \\
\hline \multirow{8}{*}{$\begin{array}{l}\text { Identificação } \\
\text { de } \\
\text { ocupação/ } \\
\text { riscos e } \\
\text { agravos }\end{array}$} & Pergunta ao usuário qual seu trabalho/ & $41(67,2)$ & $75(61,0)$ & $79(75,2)$ & $195(67,5)$ & 0,072 \\
\hline & ocupação atual & $21(34,4)$ & $53(43,1)$ & $65(61,9)$ & $139(48,1)$ & 0,001 \\
\hline & $\begin{array}{l}\text { Pergunta ao usuário quais as atividades de } \\
\text { trabalho ele desenvolve na sua ocupação atual }\end{array}$ & & & & & \\
\hline & $\begin{array}{l}\text { Pergunta ao usuário sobre o trabalho/ } \\
\text { ocupação desenvolvido por ele anteriormente }\end{array}$ & $02(3,3)$ & $20(16,3)$ & $32(30,5)$ & $54(18,7)$ & 0,000 \\
\hline & $\begin{array}{l}\text { Identifica os riscos para a saúde presentes no } \\
\text { trabalho/ocupação atual do usuário }\end{array}$ & $23(37,7)$ & $60(48,8)$ & $60(57,1)$ & $143(49,5)$ & 0,053 \\
\hline & $\begin{array}{l}\text { Identifica os riscos para a saúde no trabalho/ } \\
\text { ocupação pregressa do usuário }\end{array}$ & $10(16,4)$ & $38(30,9)$ & $43(41,0)$ & $91(31,5)$ & 0,004 \\
\hline & $\begin{array}{l}\text { Busca estabelecer a relação entre a queixa } \\
\text { e/ou doença referida pelo usuário com a } \\
\text { exposição a situações de risco para a saúde } \\
\text { presentes em seu trabalho/ocupação, atual ou } \\
\text { pregressa }\end{array}$ & $31(50,8)$ & $35(69,1)$ & $86(81,9)$ & $202(69,9)$ & 0,000 \\
\hline & $\begin{array}{l}\text { Consegue estabelecer a relação entre a queixa } \\
\text { e/ou doença referida pelo usuário com a } \\
\text { exposição a situações de risco para a saúde } \\
\text { presentes em seu trabalho/ocupação, atual ou } \\
\text { pregressa }\end{array}$ & $29(47,5)$ & $73(59,3)$ & $75(71,4)$ & $177(61,2)$ & 0,008 \\
\hline \multirow[t]{2}{*}{$\begin{array}{l}\text { Atividades } \\
\text { produtivas } \\
\text { no território } \\
\text { e agravos }\end{array}$} & $\begin{array}{l}\text { Tem conhecimento sobre a/as atividade }(\mathrm{s}) \\
\text { produtiva }(\mathrm{s}) \text { desenvolvida }(\mathrm{s}) \text { no território } \\
\text { de referência de sua unidade de saúde que } \\
\text { influencia }(\mathrm{m}) \text { a condição de saúde dos } \\
\text { usuários e da comunidade em geral }\end{array}$ & $35(57,4)$ & $84(68,3)$ & $60(57,1)$ & $179(61,9)$ & 0,160 \\
\hline & $\begin{array}{l}\text { Consegue estabelecer a relação entre } \\
\text { doenças e queixas das famílias de sua área } \\
\text { de abrangência e as atividades produtivas } \\
\text { existentes no território }\end{array}$ & $35(57,4)$ & $81(65,9)$ & $66(62,9)$ & $182(63,0)$ & 0,595 \\
\hline \multirow[t]{6}{*}{$\begin{array}{l}\text { Vigilância } \\
\text { dos agravos } \\
\text { em ST }\end{array}$} & $\begin{array}{l}\text { Conhece o Sistema de Informação de Agravos } \\
\text { de Notificação Sistema de informação de } \\
\text { agravos de notificação (SINAN) }\end{array}$ & $39(63,9)$ & $122(99,2)$ & $97(92,4)$ & $258(89,3)$ & 0,000 \\
\hline & $\begin{array}{l}\text { Sabe que algumas doenças relacionadas ao } \\
\text { trabalho são de notificação no SINAN. }\end{array}$ & $43(70,5)$ & $121(98,4)$ & $96(91,4)$ & $260(90,0)$ & 0,000 \\
\hline & $\begin{array}{l}\text { Notifica os casos de acidente de trabalho no } \\
\text { SINAN }\end{array}$ & $19(31,1)$ & $85(69,1)$ & $64(61,0)$ & $168(58,1)$ & 0,000 \\
\hline & $\begin{array}{l}\text { Notifica os casos de doenças relacionadas ao } \\
\text { trabalho no SINAN }\end{array}$ & $14(23,0)$ & $75(61,0)$ & $53(50,5)$ & $142(49,1)$ & 0,000 \\
\hline & $\begin{array}{l}\text { Realiza investigação epidemiológica dos casos } \\
\text { suspeitos notificados, para confirmação do } \\
\text { diagnóstico, fazendo relação com o trabalho. }\end{array}$ & $9(14,8)$ & $56(45,5)$ & $23(21,9)$ & $88(30,4)$ & 0,000 \\
\hline & $\begin{array}{l}\text { Elabora e implanta plano terapêutico e } \\
\text { orienta o trabalhador sobre sua situação de } \\
\text { saúde e trabalho }\end{array}$ & $16(26,2)$ & $45(36,6)$ & $49(46,7)$ & $110(38,1)$ & 0,030 \\
\hline
\end{tabular}

Fonte: Trabalho de campo realizado no município de Montes Claros, Minas Gerais, Brasil, no período de 2017/2018.

dor sobre sua situação de saúde e trabalho $(\mathrm{p}=$ $0,030)$.
A Tabela 4 apresenta a comparação do apoio técnico-especializado e institucional para de- 
Tabela 4. Comparação do apoio técnico-especializado e institucional para desenvolvimento de ações de Saúde do Trabalhador pelos profissionais da Atenção Primária à Saúde, por categoria profissional, 2018.

\begin{tabular}{|c|c|c|c|c|c|}
\hline \multirow[t]{3}{*}{$\begin{array}{l}\text { Apoio técnico-especializado } \\
\text { e institucional }\end{array}$} & $\begin{array}{c}\text { Cirurgião- } \\
\text { Dentista } \\
(n=61)\end{array}$ & $\begin{array}{c}\text { Enfermeiro } \\
(n=123)\end{array}$ & $\begin{array}{l}\text { Médico } \\
(\mathrm{n}=105)\end{array}$ & $\begin{array}{c}\text { Total } \\
(n=289)\end{array}$ & $\mathbf{P}$ \\
\hline & Sim & Sim & Sim & Sim & \\
\hline & n (\%) & n $(\%)$ & n (\%) & $\mathbf{n}(\%)$ & \\
\hline Coordenação Municipal de Atenção Básica. & $47(77,0)$ & $109(88,6)$ & $65(61,9)$ & $221(76,5)$ & 0,000 \\
\hline $\begin{array}{l}\text { Instâncias especializadas em Saúde do } \\
\text { Trabalhador. }\end{array}$ & $33(54,1)$ & $88(71,5)$ & $27(25,7)$ & $148(51,2)$ & 0,000 \\
\hline CEREST. & $43(70,5)$ & $98(79,7)$ & $38(36,2)$ & $179(61,9)$ & 0,000 \\
\hline $\begin{array}{l}\text { Teve treinamento para desenvolver ações de } \\
\text { saúde do trabalhador. }\end{array}$ & $23(37,7)$ & $87(70,7)$ & $21(20,0)$ & $131(45,3)$ & 0,000 \\
\hline
\end{tabular}

Fonte: Trabalho de campo realizado no município de Montes Claros, Minas Gerais, Brasil, no período de 2017/2018.

senvolvimento de ações de Saúde do Trabalhador, considerando as categorias profissionais. Os resultados evidenciaram que o enfermeiro apresentou resultados significativos em todas as variáveis analisadas, em relação às demais áreas: Coordenação Municipal de Atenção Básica $(\mathrm{p}=0,000)$, instâncias especializadas em ST ( $\mathrm{p}=$ $0,000)$, CEREST $(\mathrm{p}=0,000)$, teve treinamento para desenvolver ações de ST $(\mathrm{p}=0,000)$.

Sobre a articulação intrassetorial destinada a construir soluções para os problemas envolvendo as relações trabalho-saúde-doença, os resultados evidenciaram o papel importante desempenhado pelo CEREST $(60,9 \%)$ da região, seguido da Vigilância Sanitária $(39,1 \%)$ e da Vigilância Ambiental $(18,0 \%)$. Entretanto, parcela significativa dos trabalhadores entrevistados não soube informar sobre outras instâncias de articulação intersetorial $(18,0 \%)$ ou não buscou articulação $(14,9 \%)$.

\section{Discussão}

Os resultados do estudo mostram que as equipes de APS reconhecem as atividades produtivas no território e o perfil epidemiológico da população trabalhadora adscrita nesse local, entretanto, não se observaram intervenções orientadas a partir desse diagnóstico situacional. A comparação preliminar das práticas profissionais descritas apontou que os médicos se destacam na identificação do perfil epidemiológico dos trabalhadores enquanto os enfermeiros estão à frente das ações de vigilância dos agravos e do apoio técnico-pedagógico para o desenvolvimento de ações de ST.

Em relação à caracterização sociodemográfica, observou-se a predominância do sexo femini- no entre os trabalhadores da APS, confirmando outros estudos que apontam feminilização do trabalho em saúde ${ }^{20,21}$. Sobre a faixa etária, observou-se que o grupo de estudo pode ser caracterizado como jovem, na faixa etária de 31 a 40 anos $(48,8 \%)$, possivelmente refletindo as mudanças curriculares nos cursos de graduação na área de saúde que têm valorizado a capacitação profissional para atender às reais demandas do SUS, como determinado no Programa Nacional de Reorientação da Formação Profissional em Saúde (Pró-Saúde) $)^{22}$ e o Programa de Educação pelo Trabalho para a Saúde (PET-Saúde) ${ }^{23}$. Assim, os estudantes são inseridos precocemente nos cenários do Sistema Único de Saúde, sobretudo na APS, fato que pode estimular os recém-formados a buscarem oportunidade de trabalho junto ao SUS ${ }^{24}$.

O tempo de atuação na APS foi maior entre os CDs e enfermeiros que os médicos, em consonância com outros autores, os quais apontam que esta categoria tem maior grau de rotatividade, quando comparada aos outros profissionais que compõem a equipe da $\mathrm{APS}^{25}$. Em relação à formação complementar, grande parte da população estudada tinha formação específica para atuar na APS, seja por ter feito Especialização em Saúde da Família ou Residência em Saúde da Família/Medicina Família e Comunidade. O que pode ser justificado em razão de o município da realização da pesquisa ser polo do Programa de Residência Multiprofissional em Saúde da Família desde $1999^{26}$. A literatura aponta que profissionais com melhor qualificação apresentaram escores dos atributos da APS mais elevados, sendo essencial a formação específica para a consolidação da $\mathrm{APS}^{27}$. 
Dentre as ações de ST, no âmbito da APS, a PNSTT sinaliza um conjunto de ações cujo desenvolvimento cabe à equipe de saúde ${ }^{1}$. A PNAB reitera que é essencial que o processo de trabalho se configure a partir de um diagnóstico situacional do território ${ }^{28}$, indo ao encontro dos achados do estudo, nos quais os profissionais envolvidos realizavam o mapeamento das atividades produtivas, entretanto, não operacionalizavam ações de ST programadas a partir do diagnóstico situacional. Os resultados corroboram os achados de Amorim et al. ${ }^{3}$, cuja realização de ações para eliminação dos riscos associados ao trabalho foi referida por $23 \%$ dos profissionais envolvidos; e de Lacerda e Silva et al. ${ }^{14}$, os quais apontam que, apesar de os profissionais de saúde reconhecerem as repercussões do trabalho no adoecimento, apresentam dificuldade de desenvolver intervenções em ST.

Tais ações são essenciais para o reconhecimento dos potenciais riscos para a saúde dos trabalhadores e da população do território e investigação diagnóstica dos agravos e das doenças potencialmente relacionadas ao trabalho. Para tanto, faz-se necessária sua análise e incorporação no planejamento das ações de assistência, vigilância e promoção da saúde, de modo a permitir a intervenção sobre os potenciais riscos e perigos aos quais a população está exposta ${ }^{2}$.

As ações de educação em saúde voltadas para trabalhadores e a efetivação do controle social foram sinalizadas neste estudo por uma minoria de profissionais. Evangelista et al..$^{29}$, em seu trabalho com enfermeiros das Estratégias Saúde da Família (ESF), constataram que as equipes de saúde da APS realizavam ações educativas de ST, todavia, essas práticas são planejadas a partir de programas do Ministério da Saúde para população em geral e não a partir do diagnóstico do perfil produtivo no território.

Para a ST, essas ações têm o propósito de ampliar a compreensão sobre o papel do trabalho na produção do processo saúde-doença. Elas devem contribuir para o empoderamento dos trabalhadores na luta por melhores conhecimentos sobre os processos produtivos instalados no território e a influência destes na saúde das pessoas e também sobre o ambiente $e^{14,30-31}$.

Em relação às práticas de atenção à saúde dos usuários trabalhadores, segundo categoria profissional, os resultados evidenciaram que o médico identificava melhor a ocupação atual do usuário, bem como seus riscos e sua relação com os agravos. O que pode ser justificado pelo fato de caber a esse profissional, como integrante da equipe de saúde, o diagnóstico e o tratamento das doenças, segundo protocolos, diretrizes clínicas e terapêutica ${ }^{28}$. No entanto, em relação à história ocupacional pregressa, a minoria dos profissionais envolvidos no estudo, inclusive médicos, preocupava-se em fazer essa investigação. Tradicionalmente, na formação médica e dos enfermeiros, pouca ou nenhuma atenção é dada à coleta da história ocupacional, o que faz com que os profissionais, em geral, tenham dificuldades em inseri-la em suas práticas de trabalho ${ }^{31}$.

Nos achados de Amorim et al. ${ }^{3}, 26 \%$ dos investigados referiram sempre ou quase sempre perguntarem ao usuário sobre o trabalho pregresso. Silva et al. ${ }^{15}$ sinalizam que o motivo da busca de assistência pelo usuário, na maioria das vezes, não é apontado como relacionado ao trabalho. Dessa forma, não são reconhecidas pelos profissionais de saúde como problemas de ST. Nesse sentido, a coleta da história ocupacional atual e pregressa é essencial para identificar os riscos relacionados ao trabalho, auxiliar no diagnóstico e na definição do plano terapêutico, orientar o trabalhador sobre a origem do agravo, medidas preventivas, direitos trabalhistas e previdenciários e desencadear ações de vigilância em saúde ${ }^{2}$.

$\mathrm{O}$ cuidado integral à ST tem como ponto de partida o reconhecimento do trabalho e do trabalhador no território e deve identificar quem são, quantos são, quais atividades produtivas desenvolvem e em quais condições de trabalho. Esse reconhecimento acontece em vários momentos, mas, de modo geral, inicia-se na construção do diagnóstico situacional e cadastramento das famílias e continua no acolhimento, nas visitas domiciliares e na consulta, com a realização da anamnese ou história ocupacional ${ }^{2}$.

As investigações e as notificações no SINAN, atualmente, encontram-se prescritas nas portarias no 204 e no 205, de 17 de fevereiro de 2016, as quais definem a lista nacional de doenças e agravos a serem monitorados por meio da estratégia de vigilância ${ }^{32-33}$. Tal prática permite que a equipe planeje e gerencie a atenção integral aos trabalhadores. Neste estudo, o enfermeiro demonstrou ser mais efetivo quanto às ações de notificação em ST, contudo, apesar de os agravos serem notificados, a investigação dos casos suspeitos e a implementação do plano terapêutico foram ações pouco realizadas por todas as categorias profissionais, sendo o médico quem mais as elaborava e implementava. Tal prática justifica-se pelo fato de caber a essa categoria profissional a função de prover assistência a esse trabalhador com suspei- 
ta de agravo relacionado ao trabalho e estabelecer diagnóstico e tratamento ${ }^{34}$.

Os achados de Ferreira et al. ${ }^{35}$ evidenciaram que os profissionais de enfermagem que tiveram treinamentos e capacitações sobre notificação de acidente de trabalho, com 15 anos ou mais de experiência profissional, obtiveram maiores chances de fazer a notificação; e enfermeiras apresentaram quase duas vezes mais chances de notificar, quando comparadas a outras categorias profissionais do mesmo sexo.

Outros estudos corroboram com os achados e sinalizam que, apesar de a notificação de agravos em ST ser uma atividade cotidiana na APS, o caráter epidemiológico e as ações de intervenção são desconsideradas, sendo feita, na maioria das vezes, de maneira burocrática. Além do mais, ela não é reconhecida como uma ação de ST e apenas o trabalhador do mercado formal tem o registro desses agravos feito regularmente ${ }^{15,36}$.

O Caderno de Atenção Básica no 05, publicado em 2001, faz a descrição das atribuições dos profissionais da APS, em relação à ST por categoria profissional ${ }^{37}$, entretanto, ocorreram mudanças significativas nas Políticas Públicas de ST e da APS após esse período. O recém-publicado Caderno de Atenção Básica no $41^{38}$ não reforça as atribuições por categoria profissional sugerindo que todos os profissionais envolvidos na APS são responsáveis pelas ações de assistência e de vigilância.

Em relação ao apoio institucional, técnico especializado e pedagógico, o estudo sugeriu alta capilaridade e alcance junto aos enfermeiros, considerando que essa categoria profissional apontou ter apoio de todas as instâncias investigadas. O apoio da coordenação municipal de APS e do CEREST merece destaque, já que é relatado pela maioria dos enfermeiros e CDs entrevistados. Lacerda e Silva et al. ${ }^{14}$ também apontam articulação das ESFs com o CEREST para assistência em ST, ainda que de maneira pontual.

Vale destacar que uma pequena parcela de médicos (20\%) e CDs $(37,7 \%)$ afirmou ter participado de treinamentos sobre ST. Amorim et al. ${ }^{3}$, em sua pesquisa, sinalizam que $24 \%$ dos profissionais das equipes de APS indicaram ter participado de ações de qualificação e/ou educação permanente para desenvolver ações de ST. Esses achados sugerem diferentes interpretações, como falta de oferta de treinamento para esses profissionais, ausência de interesse dos profissionais envolvidos ou formação acadêmica insuficiente, evidenciando a necessidade de futuras investigações.
A literatura aponta como fragilidade dos serviços de saúde a deficiência na formação de recursos humanos no que tange à ST, tanto em nível de graduação quanto de pós-graduação; fato que pode ser evidenciado quando se avalia a produção acadêmica escassa sobre assuntos relacionados à ST ${ }^{29,39}$.

Nessa perspectiva, Mori e Naghettinni ${ }^{40}$ avaliaram a formação de médicos e enfermeiros da ESF no aspecto de ST, cujos resultados demonstram que esse campo ainda é pouco trabalhado e valorizado no meio acadêmico, não permitindo que o profissional adquira competências mínimas para atender ao trabalhador. No cenário internacional, estudos realizados na Espanha e na Itália revelam falta de treinamento dos médicos da APS em relação à saúde ocupacional, considerando a necessidade de capacitação ${ }^{10,41}$.

Para Fernandes et al. ${ }^{42}$, existe uma necessidade de rever as ementas e a forma como os conteúdos relacionados à ST são ensinados para que sejam coerentes com os projetos político-pedagógicos, pois existe um afastamento muito grande entre o que é prescrito e o que de fato é ministrado nos cursos de graduação em enfermagem. Faz-se necessária a qualificação das práticas de cuidado em ST na APS, a partir do processo de educação permanente das equipes e, sobretudo, do suporte técnico por meio do apoio matricial, considerando a necessidade de fortalecer as ações de ST na RAS ${ }^{30,41,43}$.

Apesar de os profissionais da APS estarem sobrecarregados pelas atividades assistenciais e pela demanda, quando contam com retaguarda técnica especializada e pedagógica, incorporam a ST em suas ações, fazendo com que ampliem o cuidado, sejam mais resolutivos e articulem mais com a RAS. Ademais, o apoio matricial favorece a compreensão e a incorporação do papel do trabalho enquanto determinante do processo saúde-doença ${ }^{44}$.

No tocante às limitações do estudo, destacase a não inclusão dos outros profissionais que compõem a equipe de saúde da APS na investigação, resultando em um viés de percepção em relação às práticas desenvolvidas. Outra possível limitação é que o uso de variáveis autorreferidas pode implicar em informações incorretas, embora o treinamento da equipe de campo e a realização do estudo piloto tenham sido realizados para minimizar possíveis erros da coleta.

Por outro lado, os resultados apresentam informações importantes sobre tema ainda pouco valorizado pelos profissionais de saúde - a Saúde do Trabalhador, em especial na APS. A análise de 
um grupo representativo de profissionais de saúde de um grande centro possibilitou reconhecer que os trabalhadores da APS ainda não incorporaram a ST no cotidiano de suas práxis. Os resultados contribuem para que sejam implementadas intervenções voltadas para os profissionais da APS, com o objetivo de melhorar a qualidade de assistência prestada.

\section{Conclusão}

Verificou-se que as equipes de APS incluídas no estudo realizavam o mapeamento das atividades produtivas no território e o perfil epidemiológico da população trabalhadora, entretanto, não planejavam intervenções na perspectiva da ST, a partir do diagnóstico situacional. A comparação das práticas profissionais evidenciou que o médico destacava-se na identificação do perfil epidemiológico dos trabalhadores e na implementação do plano terapêutico, enquanto o enfermeiro sobressaiu na vigilância dos agravos e no apoio técnico-pedagógico para o desenvolvimento de ações de ST. Considerando o contexto atual de mudanças no mundo do trabalho, faz-se necessária a efetiva incorporação de ações de ST no cotidiano das equipes de APS, de maneira articulada com as equipes de referência e acompanhada de intervenções que melhorem as condições de trabalho das equipes da APS, pois, apesar da prescrição legal, a inserção de ações de ST no contexto do SUS tem se configurado como um desafio permanente.

\section{Colaboradores}

DP Silva e JSB Rocha colaboraram na concepção do projeto, análise dos dados, redação do artigo e aprovação final da versão a ser publicada. RF Freitas e EC Dias colaboraram na concepção do projeto, redação do artigo e aprovação final da versão a ser publicada. LF de Souza e NA Teixeira, na coleta e tabulação dos dados, redação do artigo e aprovação final da versão a ser publicada. 


\section{Referências}

1. Brasil. Portaria $\mathrm{n}^{\circ} 1.823$, de 23 de agosto de 2012. Institui a política nacional de saúde do trabalhador e da trabalhadora. Diário Oficial da União, 2012; 23 ago.

2. Brasil. Ministério da Saúde (MS). Saúde do trabalhador e da trabalhadora. Brasília: MS; 2018. (Atenção Básica, n. 41)

3. Amorim LA, Lacerda e Silva T, Faria HP, Machado JMH, Dias EC. Vigilância em Saúde do Trabalhador na Atenção Básica: aprendizagens com as equipes de Saúde da Família de João Pessoa, Paraíba, Brasil. Cien Saude Colet 2017; 22(10):3403-3414.

4. Gomes CM, Vasconcellos LCF, Machado JMH. Saúde do trabalhador: aspectos históricos, avanços e desafios no Sistema Único de Saúde. Cien Saude Colet 2018; 23(6):1963-1970.

5. Sanson C. Debate teórico sobre o lugar do trabalho na sociedade contemporânea. Instituto Humanitas Unisinos [periódico na Internet]. 2017 Ago [acessado 2019 fev 5]. Disponível em: http://www.ihu.unisinos. br/570888-debate-teorico-sobre-o-lugar-do-trabalho-na-sociedade-contemporanea-em-edicao.

6. Organização Internacional do Trabalho (OIT). Safety and health at work. [Internet]. 2017. [acessado 2019 fev 6]. Disponível em: http://www.ilo.org/global/topics/safety-and-health-at-work/lang--en/index.htm.

7. Hämäläinen P, Takala J, Kiat TB. Global Estimates of Occupational Accidents and Work-related Illnesses. XXI World Congresson Safety and Health at Work. Singapura: Workplace Safety and Health Institute; 2017 [cited $2019 \mathrm{Fev}$ 5]. Available from: http:// www.icohweb.org/site/images/news/pdf/Report $\% 20$ Global\%20Estimates\%20of\%20Occupational\%20 Accidents\%20and\%20Work-related\%20Illnesses\%20 2017\%20rev1.pdf.

8. Observatório Digital de Saúde e Segurança no Trabalho: 2017. [Internet]. 2017 [acessado $2019 \mathrm{fev}$ 5]. Disponível em: http://observatoriosst.mpt.mp.br.

9. Santos SM. Atividade de vigilância em saúde do trabalhador no SUS [tese]. São Paulo: Faculdade de Saúde Pública, USP; 2018.

10. Riva M, Belingheri M, Castiglioni E, Bonazzi C. Atenção Primária à Saúde e Saúde Ocupacional. Resultados de uma pesquisa sobre clínicos gerais da Província de Monza e Brianza. MDL 2017; 108(1):42-51.

11. Dias MDA. Compreender o trabalho na Atenção Primária à Saúde para desenvolver ações em Saúde do Trabalhador: o caso de um município de médio porte. Rev Bras Saude Ocup 2013; 38(127):69-80.

12. Gomes EM. Análise do processo de desenvolvimento das ações de saúde do trabalhador na rede de atenção primária à saúde no Tocantins [dissertação]. Palmas: Universidade Federal do Tocantins; 2012.

13. Lacerda e Silva T, Dias EC, Ribeiro ECO. Conhecimentos e práticas de agentes comunitários de saúde na saúde do trabalhador. Interface 2011; 15(38):859870.

14. Silva T, Dias EC, Pessoa VM, Fernandes LMM, Gomes EM. Saúde do trabalhador na Atenção Primária: percepções e práticas de equipes de Saúde da Família. Interface - Comun Saude Educ 2014; 18(49).
15. Silva A, Ferraz L, Rodrigues-Junior SA. Ações em Saúde do Trabalhador desenvolvidas na Atenção Primária no município de Chapecó, Santa Catarina. Rev Bras Saude Ocup 2016; 41(e:16):1-9.

16. Vello LS. Saúde do Trabalhador na Atenção Primária à Saúde: estudo de caso em um município na região metropolitana de São Paulo [dissertação]. São Paulo: Faculdade de Saúde Pública da Universidade de São Paulo; 2015. [acessado 2019 fev 5]. Disponível em: https://www.teses.usp.br/teses/disponiveis/6/6139/tde24112015-110015/publico/LaisSoaresVello.pdf.

17. Casanova AO, Teixeira MB, Monenegro E. O apoio institucional como pilar na cogestão da atenção primária à saúde: a experiência do Programa TEIAS - Escola Manguinhos no Rio de Janeiro, Brasil. Cien Saude Colet 2014; 19(11):4417-4426.

18. Brasil. Portaria $\mathrm{n}^{\circ} 102$, de 25 de setembro de 2008 . Habilita os Centros de Referência em Saúde do Trabalhador no Estado de Minas Gerais. Diário Oficial da União. 2008; 25 Nov.

19. Amorim LA. Vigilância em Saúde do Trabalhador na Atenção Básica: contribuições para o aprimoramento das ações de saúde no município de João Pessoa, PB [dissertação]. Rio de Janeiro: Escola Nacional de Saúde Pública Sergio Arouca, Fundação Oswaldo Cruz; 2016. $150 \mathrm{f}$.

20. Costa SM, Prado MCM, Andrade TN, Araújo EPP, Silva Junior WS, Gomes Filho ZC, Rodrigues CAQ. Perfil do profissional de nível superior nas equipes da Estratégia Saúde da Família em Montes Claros, Minas Gerais, Brasil. Rev Bras Med Fam Comunidade 2013; 8(27):90-96.

21. Pinto ESG, Menezes RMP, Villa TCS. Situação de trabalho dos profissionais da Estratégia Saúde da Família em Ceará-Mirim. Rev Esc Enferm USP 2010; 44(3):657-664

22. Brasil. Ministério da Saúde (MS). Ministério da Educação. Portaria Interministerial no 2.101, de 3 de novembro de 2005. Dispõe sobre os profissionais que compõem a Comissão Executiva do Pró-Saúde. Diário Oficial União; 2005.

23. Brasil. Ministério da Saúde (MS). Secretaria de Gestão do Trabalho e da Educação na Saúde. A educação e o trabalho na Saúde: a política e suas ações. Brasília: MS; 2009.

24. Medeiros CRG, Junqueira AGW, Schwingel G, Carreno I, Jungles LAP, Saldanha OMFL. A rotatividade de enfermeiros e médicos: um impasse na implementação da Estratégia de Saúde da Família. Cien Saude Colet 2010; 15(Supl.1):1521-1531.

25. Magnaco C, Pierantoni CRA. A percepção de gestores dos municípios de Duque de Caxias e Rio de Janeiro quanto à rotatividade de profissionais na Estratégia Saúde da Família. Revista Cereus 2014; 6(1):3-18.

26. Tonelli B, Leal AP, Tonelli, W, Veloso DC, Gonçalves D,Tonelli S. Rotatividade de profissionais da Estratégia Saúde da Família no município de Montes Claros, Minas Gerais, Brasil. Rev Faculdade Odontologia - UPF [periódico na Internet]. 2018 Out. [acessado 2021 Mar.5]; 23(2):273-288. Disponível em: http://seer.upf. br/index.php/rfo/article/view/8314 
27. Leão CDA, Caldeira AP. Avaliação da associação entre qualificação de médicos e enfermeiros em atenção primária em saúde e qualidade da atenção. Cien Saude Colet 2011; 16(11):4415-4423.

28. Brasil. Portaria no 2.436, de 21 de setembro de 2017. Aprova a Política Nacional de Atenção Básica, estabelecendo a revisão de diretrizes para a organização da Atenção Básica, no âmbito do Sistema Único de Saúde (SUS). Diário Oficial da União. 2017; 21 Set.

29. Evangelista AIB, Pontes AGV, Silva JV, Saraiva AKM. A saúde do trabalhador na atenção primária à saúde: o olhar do enfermeiro. Rev Rene 2011; 12(n. esp.):10111020.

30. Dias EC, Lacerda e Silva T, Almeida MHC. Desafios para a construção cotidiana da vigilância em saúde ambiental e em saúde do trabalhador na Atenção Primária à Saúde. Cad Saude Colet 2012; 20(1):15-24.

31. Chiavegatto CV. Percepção dos profissionais de nível superior da atenção primária quanto ao desenvolvimento de ações de saúde do trabalhador no SUS em Minas Gerais [dissertação]. Belo Horizonte: Universidade Federal de Minas Gerais; 2010.

32. Brasil. Portaria $n^{\circ} 204$, de 17 de fevereiro de 2016 a. Define a Lista Nacional de Notificação Compulsória de doenças, agravos e eventos de saúde pública nos serviços de saúde públicos e privados em todo o território nacional, nos termos do anexo, e dá outras providências. Diário Oficial da União, 2016; 18 Fev.

33. Brasil. Portaria $n^{\circ} 205$, de 17 de fevereiro de 2016 b. Define a Lista Nacional de doenças e agravos a serem monitorados por meio da estratégia de vigilância em unidades sentinelas e suas diretrizes. Diário Oficial da União 2016; 18 Fev.

34. Brasil. Ministério da Saúde (MS). Secretaria de Política de Saúde. Caderno de Atenção Básica. Caderno no 5 Saúde do Trabalhador. Brasília: MS; 2002. [Internet] 2002. [acessado 2020 fev 6]. Disponível em: http://189.28.128.100/dab/docs/publicacoes/cadernos_ab/abcad27.pdf.

35. Ferreira MJM, Lima RKS, Silva AMC, Filho JGB, Cavalcanti LPG. Vigilância dos acidentes de trabalho em unidades sentinela em saúde do trabalhador no município de Fortaleza, nordeste do Brasil. Cien Saude Colet 2017; 22(10):3393.

36. Balista SRR, Silvia MS, Filho HRC. A atenção à saúde do trabalhador nas unidades básicas de saúde do SUS: estudo de caso em Campinas, São Paulo. Rev Bras Saude Ocup 2011; 36(124):216-226.

37. Brasil. Ministério da Saúde (MS). Secretaria de Políticas de Saúde. Cadernos de Atenção Básica. Caderno n5. Saúde do Trabalhador. Brasília: MS; 2002. [Internet] 2002. [acessado 2019 maio 5]. Disponível em: http://189.28.128.100/dab/docs/publicacoes/cadernos_ab/abcad27.pdf.
38. Brasil. Ministério da Saúde (MS). Secretaria de Atenção à saúde. Secretaria de Vigilância em Saúde. Saúde do trabalhador e da trabalhadora (versão preliminar). Atenção Básica, n. 41. Brasília: MS; 2018. [Internet] 2002. [acessado 2019 maio 5]. Disponível em: http:// bvsms.saude.gov.br/bvs/publicacoes/saude_trabalhador_trabalhadora.pdf.

39. Lacaz FAC, Trapé A, Soares CB, Santos APL. Estratégia saúde da família e saúde do trabalhador: um diálogo possível? Interface - Comun Saude Educ 2013; 17(44):75-87.

40. Mori EC, Naghetitni AV. Formação de médicos e enfermeiros da estratégia Saúde da Família no aspecto da saúde do trabalhador. Rev Esc Enferm USP 2016; 50(n. esp.):25-31.

41. Marguello MS, Echabe EA, Medel GT, Montrull FB, López JV. Percepcióndelpersonal médico de atención primaria de salud acerca de sus funciones, formación y conocimientosenmateria de salud laboral. Atención Primaria 2008; 40(1):7-14.

42. Fernandes MC, Souza NVDO, Mafra IF, D'Oliveira CAF, Pires AS, Costa CCP. O conteúdo da saúde do trabalhador e as metodologias de ensino na formação do enfermeiro. Esc Anna Nery 2016; 20(3):1-8.

43. Dias EC, Lacerda e Silva T. Contribuições da Atenção Primária em Saúde para a implementação da Política Nacional de Saúde e Segurança no Trabalho (PNSST). Rev Bras Saude Ocup 2013; 38 (127):31-43.

44. Lazarino MSA, Silva TL, Dias EC. Apoio matricial como estratégia para o fortalecimento da saúde do trabalhador na atenção básica. Rev Bras Saude Ocup 2019; 44(13):1-8.

Artigo apresentado em 10/03/2020

Aprovado em 20/07/2021

Versão final apresentada em 22/07/2021

Editores-chefes: Romeu Gomes, Antônio Augusto Moura da Silva 